\title{
Le interviste INN-possibili: Quando il paziente è un genio
}

\author{
di Luciano Sterpellone
}

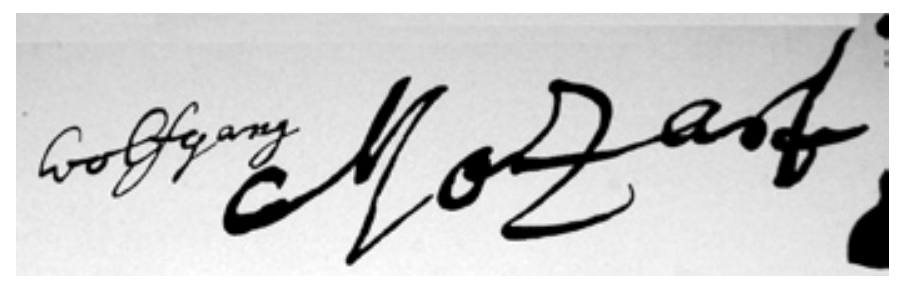

\begin{abstract}
"Una sera, un convegno sulla nefrolitiasi, una cena ove sediamo alla stessa tavola di Anna e Luciano Sterpellone. Per chi li conosce è facile immaginare la messe di argomenti con cui il Professor Sterpellone e consorte intrattengono gli astanti... sino ad arrivare all'apogeo dell'interesse e della curiosità (soprattutto di chi scrive) per la musica e $i$ compositori. Ne nasce una ridda di domande e una conversazione cosi ricca e interessante tanto che non resisto alla tentazione di chiedere al Professore di metter per iscritto, per TN\&D, una visione 'medico-storica' della malattia di Wolfgang Amadeus Mozart.”

Mi sia concesso di suggerire la lettura di questo contributo ascoltando il Requiem in re minore $\mathrm{K} 626$, almeno sino al Lacrimosa', ovvero sino a dove Mozart ha scritto di suo pugno; è ciò che il Maestro Zubin Metha e l'orchestra e Coro del Maggio hanno proposto di recente.
\end{abstract}

Marco Lombardi

Leopold Mozart, quarto violino dell'orchestra della Corte arcivescovile di Salisburgo, sorprese il piccolo Wolfgang, di appena quattro anni, mentre scarabocchiava qualcosa sulla carta: alla richiesta di che cosa stesse facendo, il piccolo rispose: «Un concerto per clavicembalo».

Non era certo un bambino abituato a dire bugie: pochi mesi dopo compose il suo primo Minuetto.

Wolfgang Amadeus era nato a Salisburgo il 27 gennaio 1756, al n. 225 dell'animata centralissima Getreidegasse, ultimo di sette figli. Il suo atto di battesimo nella chiesa di Santo Stefano reca i nomi di Johannes Chrysost(omus) Wolfgangus Theophilus, quest'ultimo (in tedesco "Gottlieb") poi latinizzato in Amadeus. Cinque anni più grande di lui era la sorellina Marianne ("Nannerl"), anche lei dotata di una musicalità eccezionale.

L'immediato periodo post partum non dev'essere stato dei migliori sia per la puerpera, frau Anna Maria, che per il neonato, entrambi "molto deboli". A quel tempo la sorte dei neonati era molto incerta, la mortalità infantile molto elevata, ed era quasi una fortuna se il bambino riusciva a superare il primo anno.

\section{Un "piccolo stregone"}

Dopo le prime notizie alquanto dettagliate sui primi momenti di vita e sulla salute del piccolo Wolfgang, per sei anni al riguardo, non si hanno più notizie su di lui. Certo è che il padre Leopold, abile violinista, non si lascia sfuggire il sorprendente talento musicale del bambino; la stessa Nannerl ricorderà che le mani del fratellino erano talmente conformate per adattarsi alla tastiera, da "non essere adatte per nessun altro lavoro".

A soli cinque anni Wolfang (tra i vari nomiglioli usati in famiglia figura anche quello di Wolfgangler, cioè "quel diavoletto di Wolfgang") fu in grado di imparare in mezz'ora un minuetto sul cembalo; a sei anni prese parte a un trio d'archi suonando la parte del violino senza aver mai studiato lo strumento: suonò così bene insieme con il secondo violinista che questi, stupito da tanta bravura, depose il violino sulle ginocchia e lasciò che il bambino suonasse da solo. Sempre a sei anni questi improvvisava piccoli pezzi, che poi il padre amorosamente trascriveva sul pentagramma.

\section{Salute, ma non troppa}

La patografia "storica" del piccolo grande musicista austriaco risale al primo viaggio che egli compì insieme con la famiglia a Vienna, e compare in una nota scritta da Leopold il 16 ottobre 1762, in cui già viene fatto cenno a problemi di tonsille del piccolo musicista.

Il viaggio a Vienna era stato accuratamente preparato da papà Leopold, con tanto di carrozza di lusso affittata per l'occasione, su cui era possibile caricare anche il clavi- 
cembalo, due violini e i bagagli. Dopo alcuni successi di pubblico raccolti a Monaco e Linz durante il viaggio, il vero exploit Wolfgang e Nannerl l'ebbero alla residenza imperiale di Schönbrunn il 13 ottobre 1762, alla presenza dell'imperatrice Maria Teresa e dei dignitari della raffinata Corte viennese.

Francesco I, colpito da tanta bravura, invitò il bambino a suonare due difficili brani con un solo dito, e poi ancora con un panno disteso sulla tastiera. Alla fine esclamò entusiasta di trovarsi davanti a "un piccolo stregone". Senza per nulla lasciarsi intimidire dall'ambiente, il piccolo "stregone" saltò in grembo all'Imperatrice, l'abbracciò e le stampò un bacio sulla guancia.

\section{Un ometto in miniatura}

Ma le giornate del piccolo Wolfgang non saranno quelle di qualsiasi bambino.

Dopo i sei anni, i suoi giocattoli saranno il violino e il clavicembalo. Del resto in casa nessuno ha interesse a distoglierlo dalla musica e lasciarlo andare in strada a giocare con i compagni; e papà Leopold (Vati) ben sa che

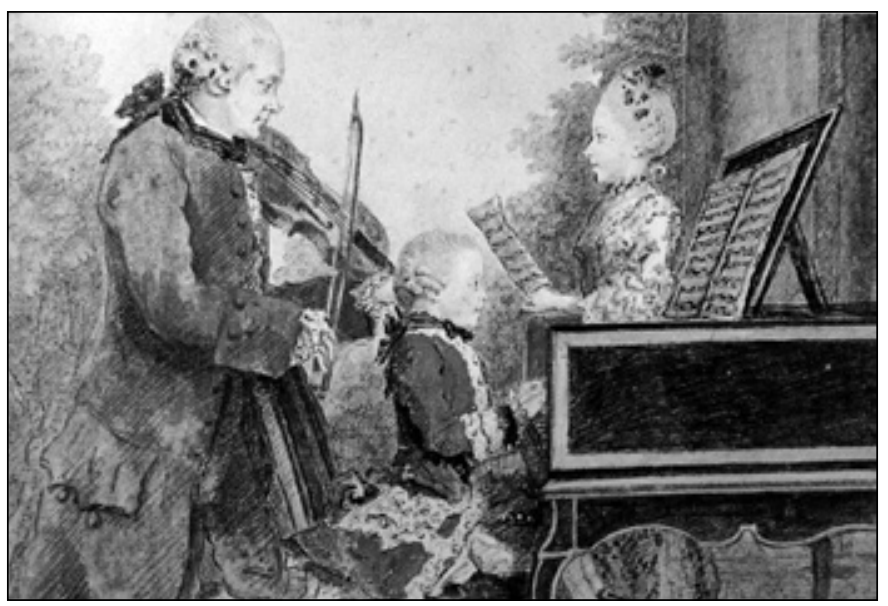

Wolfgang suona con il padre e la sorella Nannerl. Acquerello di L. Carrogis de Carmontelle (Parigi, Museo Carnavalet).

occorre sfruttare al massimo le sue capacità artistiche prima che diventi un artista adulto come qualsiasi altro. Educato a comportarsi da adulto, anche nell'aspetto Wolfgang appare un ometto in miniatura, ben acconciato con tanto di parrucca e spadino, quando - a sette anni - si incontra con Wolfgang Goethe (questi ne ha quattordici) il 18 agosto 1763 a Francoforte.

E, osservando attentamente con "occhio clinico" il piccolo Mozart nel celebre ritratto di F. L. Firmian, si nota subito la velata tristezza dello sguardo insolita per un bambino di quell' età; ma colpiscono anche alcuni caratteri somatici: le bozze frontali sporgenti, un certo buftalmo, il naso piuttosto bruttino (verrà chiamato "Mozart dall'enorme naso"), le labbra carnose con il superiore molto marcato, la mandibola sporgente e lievemente deviata verso sinistra. Qualcuno descriverà il giovane Mozart "piccolo, nasuto, dall'occhio stupido e dalla figura mediocre". Un particolare curioso è che in questo, come in altri ritratti, Mozart viene sempre ripreso di tre quarti,

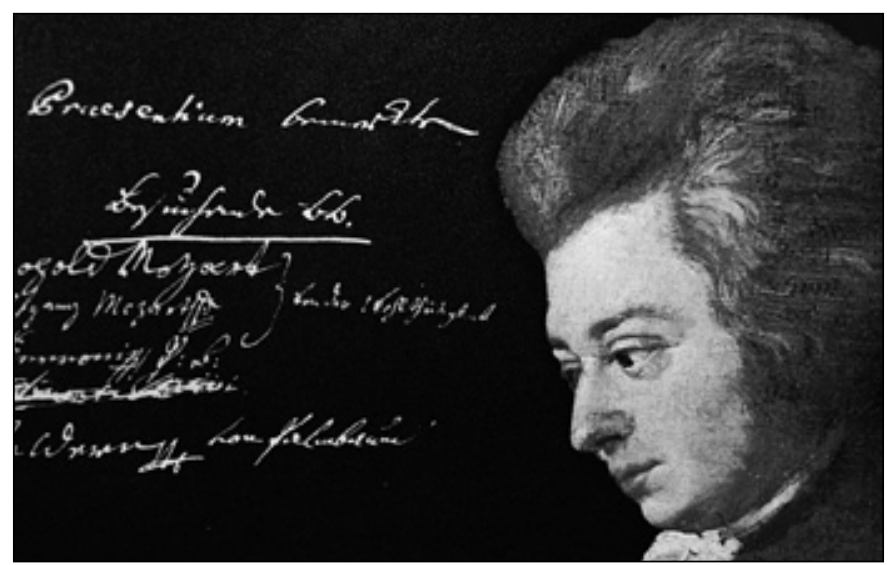

Tipico ritratto di Wolfgang Amadeus Mozart ripreso dal lato destro.

quasi a voler nascondere il suo orecchio sinistro.

Infatti questo presenta un'anomalia congenita che lo costringe a portare i capelli lunghi: appaiono difettosi il trago, l'antitrago, il lobulo e la conca, mentre il meato acustico è ristretto, stenotico. Tant'è che tutti i quadri che lo raffigurano, riprendono il musicista sempre dal lato destro: il sinistro sembra solo da intravedere, non da guardare...

Come si dirà oltre, questa malformazione verrà $a$ posteriori correlata all'esistenza di un rene policistico, che no-



Malformazione del padiglione auricolare di Mozart. 
toriamente può accompagnarsi a malformazione di altre strutture.

Ma v'è qualcosa che impedisce all'enfant prodige di essere un giovanetto sano: i frequenti episodi febbrili che lo affliggono specialmente nei viaggi, nel continuo peregrinare per l'Europa su di una traballante carrozza che, per quanto robusta e imbottita, non è certo una confortevole Roll Royce.

In realtà, poco dopo un altro trionfale secondo concerto a Schönbrunn, il bambino si mette a letto avvertendo dolori in varie parti del corpo; e lo stesso Leopold nota la presenza di chiazze sulla cute, della grandezza di un kreutzer (lo spicciolo del fiorino), rosse e rilevate, dolenti al tatto. Ma, a suo avviso, tutto dipende "dal cambiamento d'aria".

Fortunatamente la famiglia Mozart gira sempre con una piccola "farmacia da viaggio" piena di tanti piccoli intrugli, tra i quali primeggiano due "medicamenti" panacea: il Schwarzpulver e il Margrafenpulve: il primo a base di mirra, lombrichi, cuore di rana, placenta e altre sostanze disgustose, indicate caso per caso; il secondo un miscuglio di carbonato di magnesio, radice di peonia, vischio, radice di iris e polvere d'avorio.

Quale reale effetto possano avere questi "medicamenti" è assai difficile stabilire. Fatto è che dopo un paio di giorni le chiazze cutanee del promettente musicista sono divenute molto più ampie, il che crea un non lieve disappunto nell'intraprendente padre, costretto per l'occasione a disdire ogni concerto nella settimana.

Ma continua imperterrito a somministrare Pulver al malatino, anche dietro consiglio del professor Johann von Bernhard, dell'Università di Vienna, che formula la diagnosi di "esantema scarlattinoso" e prescrive una "dieta leggera”.

Oggi, a distanza di oltre due secoli, gli esperti sono del parere che si sia trattato di una forma di eritema nodoso; una diagnosi allora impossibile a farsi essendo stata questa patologia descritta soltanto nel 1860 dal dermatologo francese Antoine Bazin.

Il medico (e storico) viennese Anton Neumayr ha fatto recentemente notare che questa patologia era con ogni probabilità in rapporto all'infezione streptococcica della quale il bambino aveva sofferto qualche settimana prima a Linz; e ne avrebbe avuto una ricaduta poco dopo il suo ritorno a Salisburgo dal lungo viaggio che l'aveva portato in tutta l'Europa (1766).

Fatto è che già dopo solo due e settimane, Wolfgang poté uscire e "pagare" con un concerto le prestazioni del Prof. Bernhard. In questo concerto il bambino non si limiterà a eseguire le composizioni più complesse, ma suonerà anche un concerto per violino e accompagnerà alcune sinfonie tenendo la tastiera coperta con un panno!
In quegli anni, la salute del Wunderkind, il bambino prodigio, non è certo delle più invidiabili: il 23 settembre 1763 il bambino ha un "raffreddore poi trasformatosi in catarro". Ma non v'è tempo per fermarsi: non appena il piccolo si ristabilisce, l'intera famiglia riprende il viaggio, dapprima verso Bruxelles, il piccolo Mozart poi di qui verso la città vagheggiata da ogni artista: Parigi.

Qui, il Capodanno del 1764 l'intera famiglia Mozart viene ricevuta a palazzo reale da Luigi XV e dalla regina Maria Leszczynska.

Dopo il pranzo e i regali di rito, Wolfgang viene invitato a suonare l'organo nella cappella di Corte. Ancora una volta, applausi da spellarsi le mani. Alla fine dell'esecuzione il bambino ripete la stessa scena dell'anno prima a Schönbrunn: scende dallo sgabello dell'organo, si avvicina alla regina e, tra la meraviglia di tutti, le stampa un bel bacio sulla guancia. La nobiltà parigina farà a gara nel contendersi il Wunderkind, tant'è che per i numerosi impegni e inviti i Mozart dovranno protrarre per ben cinque mesi il soggiorno parigino.

Ma il 22 febbraio dell'anno successivo il piccolo musicista cade nuovamente ammalato. Annota il padre:

«... Questa notte il mal di gola è stato così forte che v'era pericolo che il bambino soffocasse... Anche Nannerl ha catarro, ma senza febbre... Lo sai che cosa dice qui la gente? Vogliono convincermi a inoculare il vaiolo al bambino... Io lascio il destino nelle mani di Dio, al quale solo sta far vivere questo miracolo della natura così come l'ha mandato ...»

\section{E rinuncia a variolare il figlio.}

Quei giorni si paventa infatti una recrudescenza del vaiolo, una malattia a quel tempo molto spesso mortale, che quando non uccide, lascia orrende cicatrici sul viso o su tutto il corpo. Il solo "rimedio" (in realtà molto parziale, incer to e potenzialmente dannoso) è rappresentato dalla variolazione, un metodo empirico importato inizialmente in Inghilterra - dalla Turchia - da Lady Mary Montagu, moglie dell'ambasciatore inglese a Costantinopoli, metodo poi alquanto diffuso in Europa - consistente nell'applicare in una vena del soggetto sano una piccola frazione di pus o di polvere delle croste di vaiolo umano. La millenaria esperienza popolare in Oriente ne aveva evidenziato una certa efficacia.

In Europa, il "metodo turco" cominciò ad essere accettato solo dopo che Carolina d'Ansbach, futura moglie di re Giorgio II, con...sommo spirito filantropico acconsentì a far variolare i propri figli solo dopo aver fatto sperimentare il metodo su sette condannati a morte del carcere di Newgate e su sei orfanelli di un vicino ospedale...

Da allora, la variolazione (o "variolizzazione") comin- 
ciò a diffondersi nel resto d'Europa, pur destando non poche perplessità e opposizioni. Sarà soltanto nel 1796 - cinque anni dopo la morte di Mozart - che in Gran Bretagna Edward Jenner, medico di campagna a Berkeley, perfezionò e mise a punto il suo metodo di vaccinazione "per via cutanea", poi adottato (anche se tra mille opposizioni) in tutto il mondo, utilizzando materiale proveniente non da soggetti umani come nella variolazione, ma da vacche infette (donde il nome di "vaccino").

Ma papà Leopold rifiuta di far variolare i due figli. Naturalmente, a mano a mano che Wolfgang diviene un giovanetto, la sua fama cresce parallelamente all'eco dei suoi successi. Tuttavia, dopo il lungo e trionfale successivo soggiorno londinese, la famiglia Mozart preferisce riprendere la via di casa, anche perché ormai il pubblico bada più al lato spettacolare dell'esibizione che al valore artistico dei due bambini (spesso Wolfgang si esibisce con Nannrel). Ma gli streptococchi (nessuno li aveva ancora visti!) sono sempre in agguato nelle tonsille di Wolfgang. Tant'è che, giunti a Lilla, il bambino cade di nuovo ammalato, come sarà numerose altre volte..

A posteriori, i medici che si sono interessati del "caso Mozart" hanno prospettato l'ipotesi che a causa del focolaio tonsillare cronico si sia instaurato una qualche patologia cronica sostenuta da streptococchi, "ad esempio una miocardite o una nefrite".

\section{Arriva il vaiolo}

Diagnosi a parte (a quei tempi questa riveste quasi il valore di pura e semplice esercitazione accademica, non potendo essere seguita da una terapia efficace), Wolfang si riprende nuovamente e dà altri concerti: a Monaco, a Vienna e poi in Moravia, ove nella città di Olmütz, in Moravia, viene impietosamente raggiunto dai virus del vaiolo. Con la sorella Nannrel. (Nessuno dei due è stato "variolato"):

«Alle 10 Wolfgang si lamentava agli occhi. Mi accorsi che la fronte scottava, e che le guance erano calde e rosse mentre le mani erano ghiacce. Il polso appariva più irrequieto. Gli diedi un po' di Schwarzpulver, e lo misi a dormire. Tutta la notte rimase molto irrequieto, e sentiva molto caldo. Lo portai nella camera accanto. La febbre aumentò, e gli diedi un po' di Margrafenpulver e di Schwarzpulver. Verso sera egli cominciò a delirare, e proseguì così per tutta la notte e il giorno 28. Verso sera mi recai da Sua Eccellenza il conte von Podstatsky: quando lo misi a parte che Wolfgang era malato e che prevedevo avesse il vaiolo, mi disse che ci avrebbe fatto abitare da lui, in quanto non temeva affatto la malattia. Wolfgang venne avvolto in un lenzuolo e in una pelliccia e portato alla carrozza... Appena comparvero le pustole vaiolose, la reazione febbrile sparì e il bambino non apparve più malato. Era incredibilmente pieno di pustole, ed era molto gonfio. Da ieri le pustole cadono qua e là...»

Senza togliere alcun merito al dottor Wolff che seguì il piccolo paziente sino alla guarigione, fu davvero eccezionale che questi sopravvivesse (come anche la sorella) a un'infezione come il vaiolo, allora così frequentemente mortale (a Vienna morivano nove casi su dieci). Comunque, il vaiolo lascerà i suoi segni indelebili sul visino del piccolo grande musicista, che resterà irrimediabilmente butterato per tutta la vita.

E a questo tempo che, tornato a Vienna, Wolfgang - che ha ormai dieci anni - riceve dal nuovo Kaiser Giuseppe l'incarico di comporre un'opera per la Corte viennese. Nel breve volgere di tre mesi (dall'aprile al giugno) riesce a comporre l'operina buffa in tre atti La finta semplice, che tuttavia non verrà mai rappresentata. Lì per lì il giovane se la prende a morte; ma presto si consola con un nuovo impegno: un Singspiel commissionatogli da un giovane ed eccentrico medico, il dottor Franz Anton Mesmer, che entrerà nella storia della Medicina solo come inventore di una nuova strampalata forma di cura: il magnetismo animale. Per lui Mozart compone il Bastien und Bastienne, che verrà rappresentato nel giardino d'inverno della villa dello stesso Mesmer.

\section{Tanti difetti...}

A venti anni l'immagine di Mozart non corrisponde proprio a quella tipica del grande genio musicale: è alto poco più di un metro e mezzo, piuttosto tozzo e corpulento; il viso è pallido, giallastro, butterato dal vaiolo gli occhi sono chiari, le bozze frontali sporgenti (segni evidenti di un rachitismo infantile), e le mani presentano lievi malformazioni ossee, accentuate dall'esercizio. Beethoven ne accenna nei suoi Quaderni di conversazione:

«Le dita delle mani di Mozart erano talmente arcuate dall'incessante suonare che egli non poteva nemmeno tagliarsi da solo la carne nel piatto ...»

Guardando con "occhio clinico" il celebre ritratto di F.L. Firmian, colpisce già a prima vista la velata tristezza dello sguardo del piccolo musicista, inso- 
lita per un bambino di quell'età. Ma non traspaiono ovviamente due caratteristiche psichiche che hanno contrassegnato il giovane musicista.



Mozart a 11 anni (T. Helbing, Mozarteum, Salisburgo).

La prima: un'ossessione patologica per i numeri, che condivide con la sorella e la madre.

Cinque... dieci... venti... trenta... trentasei... quarantatré...

Così conta il protagonista nella I scena de Le Nozze di Figaro mentre prende alcune misure in una stanza. No, non si tratta di numeri detti a caso: si tratta proprio di numeri ben precisi, ciascuno dei quali risulta da complicate elaborazioni numeriche di date o eventi connessi ad esperienze di vita di Mozart (sarebbe qui lungo enumerarli): ad esempio, il 9 - notoriamente multiplo di 3 - è fondamentale nei riti della massoneria, alla quale Wolfgang è affiliato.

L'altra caratteristica è un'irrefrenabile voluttà nel dire parolacce. E il pur rispettabile artista non solo si limi- ta a dire quelle comuni, ma ne inventa continuamente delle nuove aggiungendo o sostituendo lettere o sillabe alle parole, coniando così scurrilità spesso irripetibili. Pochi sanno ad esempio che egli ha titolato con espressioni scurrili due sue note composizioni: Leck mir del Arsch recht schön sauber (K 382d), e Leck mir im Arsch (K382c). Risparmiamo al lettore la traduzione dal tedesco.

$\mathrm{Al}$ riguardo va ricordato che il turpiloquio, in questo caso un'aperta "coprolalia", è uno dei caratteri eminenti della "sindrome di Gilles de la Tourette", lo psichiatra francese che la descrisse nel 1885, dalla quale secondo alcuni il nusicista era affetto.

\section{Passeggiando per il Prater}

Durante una passeggiata al Prater nella primavera del 1791, Wolfgang confessa alla moglie Costanza: «Non durerò molto. Certamente mi hanno avvelenato. Qualcuno mi ha dato dell'acqua tòfana e ha calcolato il giorno preciso della mia morte».

(L'acqua tòfana era un veleno ad azione lenta a base di arsenico, molto in voga tra il '700 e l'800, inventato da Teofania, una megera siciliana).

In realtà, da allora le condizioni di Mozart peggiorano di giorno in giorno, sempre contrappuntate da febbri e tonsilliti. Si aggravano a tal punto, che dopo la metà dell'ottobre dello stesso anno - lo costringono a letto: non si alzerà che poche volte prima di morire, pur continuando a lavorare intensamente.

Il 15 novembre egli completa la sua Cantata massonica Laut verkunde unsere Freude ("Annuncia ad alta voce la nostra gioia”). Ma dal giorno 20 la situazione precipita:

\section{«Sento che presto la musica sarà bell'e finita. Mi assale un freddo che non so spiegare».}

Gli arti sono edematosi, e il gonfiore dell'addome gli impedisce di rigirarsi nel letto. Devono cucirgli in tutta fretta una camicia da notte che s'infila dal davanti... Ma pur paventando la prossima fine, non dimentica l'impegno assunto con il conte Franz von Walsegg zu Stuppach per la Messa di Requiem: il letto di morte è cosparso di fogli di musica zeppi di note, di correzioni, di "richiami"...

Otto giorni dopo, la fine appare imminente, I medici formulano la diagnosi (buona per il tempo) di "febbre miliare infiammatoria” (hitziges Frieselfieber), che viene "trattata" con dieta blanda e ricca di liquidi, applicazione di clisteri, lassativi, emetici, sanguisughe, salassi. 


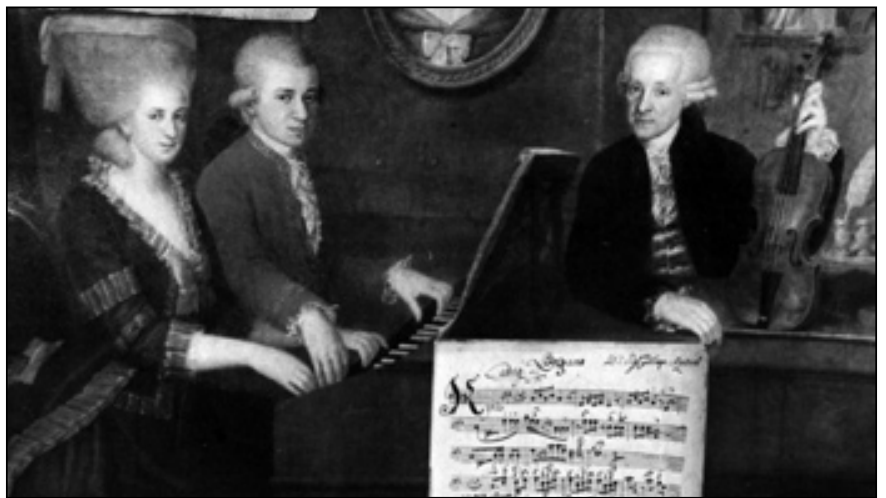

1780: Famiglia Mozart in concerto (G. Nepomuk della Croce, Mozarteum, Salisburgo).

Al farmacista verrà alla fine pagato un conto di 139 fiorini e 30 kreutzer per sudoriferi, soluzioni saline, sanguisughe ed emetici. I ripetuti salassi, praticati dal chirurgo-barbiere comunale, potrebbero aver seriamente contribuito ad accelerare la dipartita: negli ultimi dodici giorni di vita al moribondo vengono sottratti da due a tre litri di sangue...

Cinque minuti prima dell'una di notte, il grande musicista rende l'anima a Dio. Ha solo trentasei anni. Lascia non meno di seicento composizioni e... tremila fiorini di debito.

Il dì seguente, mentre un terribile temporale flagella Vienna, il suo corpo viene trasportato in tutta fretta su di un carretto al Cimitero di San Marco e depositato in una fossa comune. Ma quando il mattino dopo Costanza si reca a deporre un mazzo di fiori, la salma del marito... non si trova più. ( Né verrà mai più trovata).

Da quel giorno storici e medici si arrovelleranno intorno al "grande giallo" che portò a morte Wolfgang Mozart.

\section{Giallo per un requiem}

In realtà, pochi possono vantarsi come Mozart di aver ricevuto un numero così ragguardevole di diagnosi post mortem. I medici che lo ebbero in cura, i "testimoni oculari”, i "bene informati", i mitomani, hanno fatto a gara nel gettare lo scompiglio tra le già confuse notizie di cui si disponeva sugli ultimi giorni del compositore, oltretutto imprecise e frammentarie per la penuria di documenti diretti e per la terminologia fantasiosa, oggi in gran parte obsoleta, con cui si indicavano certi mali.

La palma d'oro della fantasia va senz'altro allo scrittore russo Puskin, che nel 1831 (quaranta anni dopo la morte di Mozart) scrisse un testo drammatico intitolato Mozart e Salieri in cui - raccogliendo le voci che giravano per Vienna - sosteneva che Mozart fosse stato avvelenato dal Antonio Salieri, un musicista molto quotato al tempo, suo aspro rivale. La "favola metropolitana" divenne poi "realtà" nell'immaginario comune dopo che il testo di Puskin, musicato (1898) da Rimsky-Korsakoff, fu portato al grande pubblico. Nei decenni successivi, demolita definitivamente dagli storici una teoria così fantasiosa (ma non ancora spenta del tutto), ci si è orientati verso una ricerca più seria e documentata nel tentativo di chiarire il perché Mozart sia morto a soli 36 anni.

Attualmente molti studiosi sostengono che la malattia terminale di Mozart sia stata un' "insufficienza renale" secondaria a nefrite cronica, evoluta in una pielonefrite e sfociata in una "nefrosclerosi pielonefritica”. Le "coliche" di cui parla il padre Leopold potrebbero essere state crisi dolorose collegate a questa patologia.

Ora, senza addentrarci nei particolari delle teorie, basta dire che, sfrondando le ipotesi decisamente improbabili, la rosa delle possibili cause si restringe praticamente a: 1) malattia renale con coma uremico terminale; 2) influenza (in quel periodo infuriava in Austria una grave epidemia influenzale; 3) febbre reumatica acuta; 4) eccesso di salassi. L'ipotesi privilegiata resta comunque quella di una compromissione renale, favorita, da tre fattori: focolaio streptococcico tonsillare (che ha agito sin dai primi anni di vita); azione del tetracloruro di mercurio costituente il "liquore di van Swieten", un "ricostituente" alla moda inventato dal medico dell'Imperatrice e di cui il musicista si serviva regolarmente, che avrebbe agito come tossico su un suo rene, probabilmente policistico.

Quest'ultima ipotesi "genetica” è stata prospettata nel 1957 da due urologi italiani, R. Cacchi e A. Marini: sarebbe esistita una malformazione congenita del rene, nella fattispecie di un rene policistico. La morte sarebbe sopravvenuta in seguito all'insufficienza renale frequente in questa patologia.

Anche il Professor A.E. Rappaport, eminente patologo della Florida e acceso ammiratore di Mozart, riprendendo le osservazioni di numerosi studiosi statunitensi e svizzeri secondo le quali molte malformazioni dei reni e delle vie urinarie si accompagnano ad anomalie congenite dell'orecchio, ha prospettato la possibilità che nel caso di Mozart la nota malformazione dell'orecchio sinistro (vedi prima) fosse associata a un'anomalia renale.

La sua teoria trova conforto in un passo della biografia di Nissen, che alla pagina 586 così dice: 
«I lineamenti del viso e l'orecchio del figlio di Wolfgang sono simili a quelli del padre. Ciò che più colpisce è la conformazione dell'orecchio di Mozart, del tutto diverso dal normale, e che soltanto il secondo figlio ha da lui ereditato».

È curioso che una notizia del genere si trovi, insieme al disegno dell'orecchio sinistro di Mozart e di quello del figlio, dietro insistenza di Costanza, in questa famosa biografia redatta dal suo secondo marito nel 1829. Le voci secondo le quali il suo secondo figlio sopravvissuto, Franz Xavier Wolfgang, fosse frutto della relazione adulterina con Süssmeyer (anche lui si chiamava Franz Xavier...) erano di fatto ancora molto insistenti a quel tempo: ora, dichiarando che anche il figlio presentava questa stessa anomalia, in particolare la "mancanza della conca auricolare", Costanza dava intrinsecamente la prova che il padre non potesse essere che Mozart.

Chissà, approfittando del fatto che nessuno sapeva ancora nulla di DNA, era questo per lei un ottimo alibi per nascondere qualche scappatella... 